Revue internationale P.M.E.

Économie et gestion de la petite et moyenne entreprise

Revue

internationale

PME

\title{
De la petite entreprise au groupe
}

\section{Aldo Enrietti}

Volume 2, numéro 2-3, 1989

URI : https://id.erudit.org/iderudit/1007935ar

DOI : https://doi.org/10.7202/1007935ar

Aller au sommaire du numéro

Éditeur(s)

Presses de l’Université du Québec

\section{ISSN}

0776-5436 (imprimé)

1918-9699 (numérique)

Découvrir la revue

Citer cet article

Enrietti, A. (1989). De la petite entreprise au groupe. Revue internationale P.M.E., 2(2-3), 201-210. https://doi.org/10.7202/1007935ar

\section{Résumé de l'article}

Le but de cet article est d'exposer les formes à travers lesquelles se structure la petite et moyenne entreprise italienne : en fait, si la représentation traditionnelle est celle d'un système productif très fragmenté, dans la réalité il y a une pluralité de structures organisationnelles (les districts, les constellations, les groupes, les réseaux) qui mettent en évidence l'existence soit de formes structurées d'aggrégation, soit des rapports d'interdépendance et de collaboration entre différentes entreprises. d'utilisation que vous pouvez consulter en ligne.

https://apropos.erudit.org/fr/usagers/politique-dutilisation/ 


\title{
De la petite entreprise au groupe
}

Aldo ENRIETTI

Université de Turin

\begin{abstract}
RESUME
Le but de cet article est d'exposer les formes à travers lesquelles se structure la petite et moyenne entreprise italienne : en fait, si la représentation traditionnelle est celle d'un système productif très fragmenté, dans la réalité il y a une pluralité de structures organisationnelles (les districts, les constellations, les groupes, les réseaux) qui mettent en évidence l'existence soit de formes structurées d'aggrégation, soit des rapports d'interdépendance et de collaboration entre différentes entreprises.
\end{abstract}

\section{ABSTRACT}

The aim of this article is to show that the small and medium italian firms are is several forms that get over the signle firm; in fact, the traditional representation of the italian industry as very fragmented does not size the existence of organizational structures (districts, constellations, groups, networks) emphasizing both structural forms of aggregation, interdependance and collaboration relationships between small and medium firms.

\section{RESUMEN}

El fin preciso de este articulo es de exponer las formas atravez de las cuales se estructuran la pequena y mediana empresa italiana; de esta forma, si la representacion tradicional es la de un sistema productivo bastante fragmentado, en la realidad existe una pluralidad de estructuras organizacionales (los distritos, las constelaciones, los grupos, las redes) que ponen en evidencia la existencia ya sea de formas estructuradas de agregacion o de relaciones de interdependencia y de colaboracion entre diferentes empresas.

- Aldo Enrietti est chercheur en économie industrielle au département d'économie de l'Université de Turin. Adresse: Dipartimento di Economia - Via S. Ottavio, 2010100 Torino. 


\section{Introduction}

Le but de cet article est d'exposer quelques unes des formes de structure de la petite et moyenne entreprise en Italie; en supposant qu'au delà d'une image d'un système productif très fragmenté se manifeste une pluralité de structures organisatives (le district, la constellation, les groupes, les réseaux) qui dépassent la dimension de chaque entreprise.

L'importance et la différence des petites entreprises italiennes par rapport aux grands pays européens en est le point de départ (tableau 1).

\section{Tab. 1 Répartition de l'emplol par tallie d'entreprise dans l'industrie manufacturière}

\begin{tabular}{|lrrrr|}
\hline & Italie & $\begin{array}{r}\text { France } \\
\%\end{array}$ & Allemagne & Grande-Bretagne* \\
& & 26,4 & 18,6 & \\
\hline$<100$ & 59,2 & 41,7 & 29,1 & 19,1 \\
$100-400$ & 21,2 & 31,9 & 53,3 & 34,4 \\
$>500$ & 19,6 & & & 46,5 \\
\hline
\end{tabular}

* $\quad 1981$ P 1982

\section{Interdépendance et collaboration : le district industriel}

Les statistiques italiennes plus anciennes montraient un système industriel polarisé entre grandes et petites entreprises, celles-ci étant le plus souvent fortement dispersées et subordonnées aux grandes. Or, les recherches des années 70 et $80^{1}$ ont au contraire contribué à mettre en évidence différentes formes d'agrégation territoriales des entreprises (les zones, les districts industriels...) comme élément fondamental de ce système, au-delà de la répartition grande/petite entreprise.

1. En particulier, Bagnasco (1977), Brusco (1982), Garofoli (1981 et 1982), Varaldo(1979), Fuà et Zacchia (1983). 
Parmi les divers éléments caractéristiques de ces différentes appréciations, nous voulons souligner un système présentant une forte interdépendance et une collaboration à l'intérieur d'un contexte de concurrence. C'est le cas des districts industriel $\mathrm{s}^{2}$ constitués par un grand nombre de petites entreprises spécialisées dans chaque phase d'une même production et regroupées dans une même zone industrielle. Ces différentes entreprises qui produisent le même produit fini ou partiellement fini, ou qui le compose, sont en concurrence entre elles; mais elles font partie d'un système social dans lequel la coopération qui transcende les rapports économiques est devenue une habitude

Le district est donc caractérisé par cette intégration réciproque de concurrence et de coopération, où le premièr élément pousse à des efforts continus pour économiser les ressources et trouver toutes les innovations utiles pour augmenter le rendement. A son tour la coopération peut être vue sous divers aspects : d'abord elle constitue un facteur de réduction des risques pour celui qui entreprend une nouvelle activité ou décide de nouveaux investissements; cela représente une sorte de réseau de protection social pour l'entrepreneur qui, en cas d'insuccès, peut rester dans le même milieu en travaillant pour d'autres personnes au lieu de travailler pour son compte; cette assurance permet l'augmentation du nombre de ceux qui sont disposés à participer à la compétition. Ensuite, puisque le district représente un ensemble d'activités étroitement complémentaires ${ }^{3}$ l'exigence d'une coordination soit qualitative soit quantitative peut être satisfaite grâce à une coopération réciproque qui joue le rôle d'une espèce de «main visible».

En outre, la coopération contribue à faire baisser les coûts de production des entreprises en leur permettant de profiter des économies externes (d'échelle, de coordination, de qualification du produit, de formation du personnel...) qui dérivent de l'existence du district dans son ensemble. Enfin, la coopération limite les coûts de transaction (Williamson, 1985). En effet, «dans le district industriel, l'opportunisme est privé d'encouragement; sans recourir à des contrôles coûteux ou à des contrats très complexes. En fait, face à l'incertitude, la flexibilité et l'innovation dans ces districts tendent à favoriser une demande finale diversifiée plutôt que standardisée; l'ambiguité est dépassée grâce à la décomposition de l'entreprise, plus qu'à travers des règles bureaucratiques de coordination confiées à des managers salariés» (Dei Ottati, 1987 : 140).

2. Becattini (1988).

3. Pour une analyse poussée des rapports de complémentarité entre les entreprises, voir Richardson (1972). 


\section{Une constellation d'entreprises}

Avec ce concept de district industriel, il est possible d'opérer une recomposition des activités des petites entreprises. Mais un problème se pose ici : le district représente un système d'entreprises à mailles trop larges lorsque l'on veut analyser d'une façon plus spécifique les petites et moyennes entreprises appartenant à une combinaison bien déterminée de produit-marché-technologie. Dans ce cas il est nécessaire de se référer à une unité d'enquête qui se place à un niveau intermédiaire entre la petite entreprise et le système plus vaste du district.

Un premier cas - typique du district - concerne le rôle exercé par les agent spécialisés dans la commercialisation. Les petits entrepreneurs liés entre eux, orientés vers l'activité de gestion du processus productif, ne sont pas en mesure ni de connaître à fond les marchés d'approvisionnement et de débouchés, ni de disposer d'informations sur l'évolution de la demande en général, sur les politiques économiques, sur l'évolution des marchés des valeurs. Ces tâches sont au contraire exercées par des entreprises spécialisées dans la commercialisation. Elles s'occupent donc de l'achat des matières premières ou autres «inputs» en dehors du district, ainsi que de la vente et, quelquefois même, du design des produits. Il se crée donc un lien entre une entreprise de services, ayant un rôle de leader, et un groupe de petites entreprises manufacturières pour ce qui touche leurs débouchés sur le marché.

Un autre exemple de ce niveau intermédiaire est la «constellation» caractérisée par un nombre limité d'entreprises (5 à 10) qui se divisent le travail et opèrent selon des rapports d'interdépendance réciproque en ce qui concerne la production d'un produit complexe ou final; certaines de ces entreprises sont engagées dans des activités intermédiaires ou complémentaires, d'autres interviennent sur des phases terminales (Lorenzoni, 1983 : 219).

L'élément principal de cette constellation est l'existence d'une «entreprisemaître-d'oeuvre», souvent celle qui assure la phase terminale, à laquelle les autres entreprises se positionnent et s'adaptent. Les rapports entre les entreprises de la constellation peuvent être soit informels, c'est-à-dire de nature non contractuelle, soit formels lorsque «l'entreprise-maître-d'œeuvre» possède une participation partielle ou majoritaire dans le capital des autres entreprises. Mais il ne s'agit pas encore d'un vrai groupe car il manque une homogénéité de langages, de transmission de compétence de type managérial, d'une gestion unitaire des ressources humaines et financières et une politique commune de bilan.

Enfin la forme plus récente de ce niveau intermédiaire d'organisation pour les petites et moyennes entreprises est le «groupe» dont la diffusion est étroitement liée aux tranformations intervenues dans les années 80 . 


\section{Les nouvelles caractéristiques de la concurrence}

Pendant la deuxième moitié des années 70, les entreprises de petite dimension ont représenté la partie la plus dynamique des structures productives de tous les principaux pays industrialisés et ont joué un rôle important dans la création de nouveaux emplois, dans la flexibilité des systèmes productifs et dans le maintien de la concurrence.

Avec les années 80 , certains phénomènes, en particulier l'accélération du progrès technologique et la globalisation progressive des marchés, semblent mettre en évidence les désavantages de la petite dimension par rapport à la grande.

L'activité innovative est un des problèmes les plus importants à résoudre pour les petites et moyennes entreprises. Pendant qu'elle devient un facteur de plus en plus important pour la compétition sur les marchés, les tendances scientifiques et technologiques en rendent plus complexe et problématique l'adoption pour la petite entreprise. En outre, l'accumulation de compétences innovatrices devient de plus en plus importante dans le secteur de la structure organisative, informative, de gestion et de promotion.

Certains éléments de la situation italienne font bien ressortir ce problème. En premier lieu, de récentes analyses de l'ISTAT et de l'Ecole Polytechnique de Milan sur la diffusion de systèmes d'automation intégrés en Italie ${ }^{4}$ montrent que de telles applications proviennent spécialement des grandes entreprises. Ensuite, dans les petites et moyennes entreprises, l'importance relative de l'activité d'innovation est assez faible, surtout la R\&D où l'innovation correspond à un processus d'apprentissage grâce à l'expérience.

La tendance à la globalisation des marchés demande aux entreprises de structurer leur présence à l'étranger de façon stable, au moyen d'importants investissements sur des marchés spécifiques et avec souvent une capacité d'accord et d'intégration avec d'autres entreprises. Le système des petites et moyennes entreprises italiennes a jusqu'ici montré une capacité de compétition particulière sur les marchés étrangers; le résultat est que les entreprises de moins de 300 employés détiennent $40 \%$ du total des exportations manufacturières (Ministère de l'Industrie, 1989 : 143).

L'aspect négatif de cette bonne performance en matière d'exportation est la forte fragmentation de l'offre. En 1985, environ un tiers des exportations italiennes provenaient d'entreprises ayant un chiffre d'affaire inférieur à 5 milliards de lires; en 1983, environ deux tiers des entreprises exportatrices fonctionnaient sur un seul marché (Ministère de l'Industrie, 1989 : 44). Il est évident que la petite dimension peut avoir certains handicaps parmi lesquels des difficultés à dépasser les barrières des marchés lointains, d'autres, d'investir pour consolider l'expertise ou une moindre possibilité d'accès au marché financier, ou enfin, une plus grande instabilité dans leur présence sur les marchés étrangers.

4. E. Pagnotta (1989), «L'innovazione non ama il piccolo», Mondo Economico, 14 janvier; S. Mariotti (1989), «Più flessibili con l'high tech», Mondo Economico, ler avril. 


\section{$5 \quad$ Les groupes d'entreprises}

Dans le nouveau contexte compétitif et technologique, le groupe se présente comme une stratégie de croissance des PMI, capable d'affronter les questions discutées précédemment.

Deux récentes recherches en Italie ont mis en évidence la diffusion de ce phénomène. Ainsi, de l'analyse de 2574 opérations d'achats réalisés en Italie entre le 1er janvier 1983 et le 31 décembre 1987 (Bianchi, Gualtieri, Pancaldi et Sassatelli, 1988) il résulte que les entreprises de petite et moyenne dimension ont joué un rôle particulièrement actif sur les marchés. Ainsi, environ 30\% des entreprises acheteuses ont moins de 500 employés et $8 \%$ moins de 20 employés; en outre, si on considère non seulement la taille de chaque entreprise acheteuse mais aussi celle du groupe de contrôle, il résulte que les entreprises indépendantes ou appartenant à des groupes avec un chiffre d'affaire inférieur à 500 milliards de lires ont effectué environ $45 \%$ de toutes les opérations considérées, contre $31 \%$ réalisées par la plupart des autres groupes italiens et $24 \%$ conclues par des groupes à capital étranger. On peut donc en déduire que le processus de concentration au moyen d'acquisitions entraîne aussi le dynamisme des entreprises plus petites (Bianchi et al., 1988 : 190). Du reste, le fait même que les groupes plus petits soient impliqués dans cette politique est cohérant avec les résultats d'autres recherches (Arrighetti, 1989). Les secteurs à plus faible concentration où il existe une plus grande diffusion des petites et moyennes entreprises (textile, aliments, habillement, peaux et cuir, bois et meuble) sont ceux où la concentration a le plus augmenté durant cette période, en particulier avec des entreprises-maître-d'oeuvre; la plupart des petits groupes sont fortement représentés dans ces secteurs.

Par conséquent, des petites et moyennes entreprises dans les années 70 sont devenues des groupes de moyenne/grande dimension grâce à une forte croissance interne et externe qui leur a permis de gagner des quotes-part de marché. Même dans les investissements directs à l'étranger, les entreprises ou les groupes mineurs ont été présents d'une façon significative, en moyenne dans $40 \%$ des cas.

Ainsi, l'analyse des données par secteur semble montrer que les petites entreprises utilisent la croissance externe de façon essentiellement horizontale pour obtenir des économies d'échelle liée au volume de production de produits spécifiques.

L'implication de petits groupes dans le processus de croissance externe représente une situation à laquelle il faut prêter attention. En premier lieu, parce que cette implication permet à certaines entreprises d'être partie active du processus de restructuration et de changement du système productif italien. En second lieu, parce qu'elle met en évidence le recours de plus en plus fréquent à des configurations de groupes ou à des structures organisationnelles typiques des grandes entreprises qui se répandent même parmi les entreprises plus petites (Bianchi et al., 1988: 208-209). 
Une recherche sur ce phénomène des petits groupes a été faite en Lombardie auprès de 35 groupes industriels à capital national et avec un chiffre d'affaire entre 10 et 200 milliards (IRER, 1988).

Tab. 2. Formation des groupes en Italie

\begin{tabular}{|l|r|r|r|r|}
\hline \multirow{2}{*}{ Période } & \multicolumn{4}{|c|}{ Nombre de sociétés } \\
\cline { 2 - 5 } & filiales & regroupements & \multicolumn{1}{|c|}{ total } & \% regroupements \\
\hline jusqu'en 1976 & 22 & 6 & 28 & 21,4 \\
$1976-1980$ & 39 & 9 & 48 & 18,8 \\
après 1980 & 18 & 26 & 44 & 59,1 \\
\hline TOTAL & 79 & 41 & 120 & 34,2 \\
\hline
\end{tabular}

En examinant la période où les entreprises ont pris l'initiative de former un groupe (tableau 2), on observe que les acquisitions se concentrent nettement dans les années suivantes à $1980(59,1 \%)$; tandis que dans les années précédentes, on voit des créations de filiales encouragées par les facilités fiscales du moment. On peut soutenir que la «filiation» représente la phase initiale et expérimentale du développement du groupe. Cette formule par la suite prend de l'importance en utilisant les mêmes mécanismes et la même façon de diriger.

Dans ce cas, il ne s'agit pas de groupes industriels au sens propre du terme... Mais on a affaire : a) à des maisons-mère entourées de satellites auquels est confié uniquement la gestion des activités manufacturières; b) à des petits groupes d'entreprises opérant de façon autonome et constitués par des entreprises controllées par un seul agent économique à l'aide de participations personnelles (IRER 1988 : 187).

Au contraire, le regroupement suppose en général des objectifs nets et stratégiques, une délimitation du rayon d'action des entreprises, une capacité d'homogénéisation dans la façon de diriger, et une culture de l'entreprise, tous facteurs qui proviennent d'une expérience consolidée. Du reste, la crise économique du début des années 80 a offert plusieurs opportunités d'acquisition d'entreprises efficaces du côté de la production, mais en crise financière ou incapables d'exploiter d'une manière adéquate leur propre potentialité.

La recherche de l'IRER (1988: 195) souligne aussi l'existence d'un parcours évolutif entre différentes structures organisationnelles grâce au passage d'une vision élémentaire de l'organisation (petits groupes d'entreprises) à un vision plus large et plus structurée (le groupe) avec laquelle se réalisent des conditions efficaces de direction et d'activités assez complexes d'innovations et de développement. 
En reprenant donc nos arguments, on peut dire que le passage de l'entreprise individuelle au groupe devient une des conditions à l'intérieur d'activités considérées assez solides pour affronter la concurrence et les défis de la nouvelle technologie, sans constituer toutefois l'instrument pour assumer une nette position de leadership sur le marché ou dans les technologies.

\section{Les réseaux d'entreprises}

Toutefois, pour être complète, l'analyse de l'évolution des groupes de petite dimension doit tenir compte de deux aspects assez importants.

En premier lieu les analyses discutées ici se réfèrent à un "groupe formel» constitué par des entreprises liées entre elles par des rapports de propriété. En réalité, très souvent, autour de ce noyau central, il existe aussi un «groupe informel» formé d'un ensemble d'entreprises externes et, du point de vue juridique, autonome; mais du point de vue productif ces entreprises sont étroitement liées à l'activité du «groupe formel», étant donné que la totalité ou la plus grande partie de leur chiffre d'affaire lui est destiné. Il s'agit d'une situation appelée quasi-intégration verticales.

Par conséquent, le groupe formel lie des entreprises qui s'occupent des fournitures sans toutefois encourir des obligations de type organisationnel et financier qui se rencontrent quelquefois dans le cas d'un investissement direct profitant de possibilités importantes de flexibilité.

Un cas typique d'une structure de ce genre est la Benetton ${ }^{6}$ qui, en centralisant les fonctions stratégiques, met en adjudication des phases de son processus de production à quelques centaines d'entreprises extérieures dont les rapports désormais sont formalisés. En plus des entreprises manufacturières, la Benetton a réalisé une structure de type réseau dans la distribution grâce au système de franchise. Cela signifie un élément de plus dans le réseau réalisé par la Benetton par rapport au système traditionnel de décentralisation, et aussi un meilleur emploi des technologies de l'information servant à mieux relier les diverses unités. On trouve donc un réseau télématique mondial pour les commandes, l'automation du magasin central et la planification de l'adjudication des contrats, de façon à produire le maximum d'efficacité de production et la plus grande adéquation avec les différents marchés.

Avec le réseau d'entreprises on passe donc à une structure organisationnelle qui - conceptuellementet opérationnellement — va au delà tant du district industriel que du système industriel, reliant horizontalement toutes les réalités du territoire et

5. Blois (1972), Enrietti (1983).

6. Nardin (1986). 
tous les secteurs. Jusqu'à maintenant le groupe a été présenté comme un sujet économique autonome dans un rapport de domination par rapportà son propre réseau d'entreprises; cette vision est pourtant limitée dans la réalité du système industriel italien et ne prend pas en considération l'appartenance des groupes ou des réseaux à un plus grand réseau mis en oeuvre par une grande entreprise ou par un grand groupe, par exemple FIAT ou OLIVETTT ${ }^{7}$. La tendance est la constitution de groupes de petites et moyennes dimensions à l'intérieur d'un réseau d'entreprises dominé par une grande ${ }^{8}$. Elle possède des spécificités qui peuvent être analysées en partant de l'exemple de la filiale automobile et du rôle joué par la FIAT'.

Dans les années 70, dans l'industrie automobile (Altshuler et al., 1984), on assiste à un déplacement de la concurrence par l'innovation technologique et par tous les aspects touchant la qualité, la confiance, la durée, le confort des voitures. Cela demande à l'industrie automobile d'investir les ressources nécessaires pour faire face aux innovations. Le rôle des fournisseurs subit un profond changement qui leur attribue une fonction de première importance dans le processus d'innovation.

Les caractères de collaboration et de coopération à l'intérieur de la filiale se sont donc accentués, en particulier avec les entreprises capables de répondre aux exigences d'innovation. Les entreprises deviennent responsables de systèmes fonctionnels entiers plutôt que de simples détails. Il s'agit du développement de la politique de partenariat ${ }^{10}$. Un nombre limité d'entreprises développe donc un rôle d'interlocuteur privilégié avec les industries automobiles.

Ainsi, dans le cas de la FIAT, le nombre d'entreprises-maître-d'oeuvre, responsables des projets, de l'approvisionnement, de l'assemblage, des essais de sous-ensemble ou de fonction est d'environ 250. Il s'agit de fournisseurs de premier plan qui doivent affronter un réseau de leurs propres fournisseurs (de second niveau). Ce passage de la fourniture simple à celle du sous-ensemble a besoin, pour les petites entreprises, d'une structure formelle ou informelle, pour faire face d'une manière adéquate aux exigences d'innovation et de qualité imposées par les industries automobiles.

Bref, le passage à une analyse de réseau met en évidence l'évolution de la structure à l'aide de cercles concentriques entre les entreprises qui sont à la tête des grandes : un premier cercle autour de la grande entreprise avec les fournisseurs leaders, un deuxième cercle dans lequel se situent les fournisseurs de second niveau. Il faut donc souligner le rôle actif des grandes entreprises dans la transformation des petites et moyennes vers une structure organisationnelle plus complexe.

7. Le thème des grands groupes italiens à être développé par Alzona (1988 et 1989).

8. C'est la thèse aussi de Sabel (1988).

9. Pour une analyse détaillée de la structure de Fiat, voir Enrietti et Fornengo (1989).

10. de Banville (1989). 


\section{BIBLIOGRAPHIE}

Altshuler, A., Anderson, M., Jones, O., Roos, D. et Womack, J., (1984), The Future of Automobile, George Allen \& Unwin, Londres.

Alzona, G., (1988), «Acquisizioni a cessioni nella dinamica dei grandi gruppi industriali italiani», dans Bianchi.

Alzona, G., (1989), «Logiques d'implantation sectorielle des groupes de sociétés : una comparaison Italie-France», Revue d'Economie Industrielle, $\mathrm{n}^{\circ} 47$.

Arrighetti, A., (1989), «Forme di mercato e dinamica della concentrazione industriale», dans Paddan Pezzoli et Silva.

Bagnasco, A., (1977), Tre Italie, Il Mulino, Bologne.

Banca d'Italia, (1988), «Atti del seminario : Ristrutturazione economica e finanziaria delle imprese», Rome.

de Banville, (1989), «Le développement du partenariat industriel», dans Revue d'Economie Industrielle, $\mathrm{n}^{\circ} 47$.

Becattini, G., (1987), (éd.), «Mercato e forze locali : il distretto industriale», Il Mulino, Bologne.

Bianchi, P., (1988), (éd.), «Antitrust e gruppi industriali». Il Mulino, Bologne.

Bianchi, P., Gualtieri, G., Pancaldi, A. et Sassatelli, M., (1988), «Determinanti delle acquisizioni in Italia», dans Banca d'Italia.

Blois, K. J., (1972), «Vertical Quasi Integration», Journal of Industrial Economics, juillet.

Brusco, S., (1982), «The Emilian Model : Productive Decentralisation and Social Integration»,Cambrdige Journal of Economics, $\mathrm{n}^{\circ} 6$.

Dei Ottati, G., (1987), «Il mercato comunitario», dans Becattini.

Enrietti, A., (1983), «Industria automobilistica : la «quasi integrazione verticale» come modello interpretativo dei rapporti tra imprese», Economia e Politica Industriale, $\mathrm{n}^{\circ} 88$.

Enrietti, A. et Fornengo, G., (1989), «Il gruppo Fiat», La Nuova Italia Scientifica, Rome.

Fuà, G. et Zacchia, C., (1983), (éd.), Industrializzazione senza fratture, Il Mulino, Bologne.

Garofoli, G., (1981), «Lo sviluppo delle aree periferiche nell'economia italiana degli anni settanta», L'industria, $\mathrm{n}^{\circ} 2$.

Garofoli, G., (1988), «Le aree-sistema in Italia», La Politica en Economia, $n^{\circ} 11$.

IRER, (1988), «L'innovazione organizzativa nell'industria minore. Lo sviluppo per gruppo industriale», Franco Angeli, Milano.

Lorenzoni, G., (1983), «La costellazione di imprese : una base di indagine sui processi di sviluppo», Economia e politica industriale, $\mathrm{n}^{\circ} 38$.

Ministero dell'industria, (1989), «Rapporto della Commissione per lo studio delle problematiche delle piccole e medie imprese», l'Industria, $\mathrm{n}^{\circ} 1$.

Nardin, G., (1986), «Benetton : strategia e struttura di un'impresa di successo», Edizioni Lavoro, Roma.

Paddan, P. C., Pezzoli, A. et Silva, F., (1989), (éd.), «Concorrenza e concentrazione nell'industria italiana», Il Mulino, Bologne.

Richardson, G. B., (1978), «The organization of industry», Economic Journal, $n^{\circ} 82$.

Sabel, C.F., (1988), «La nuova rilevanza delle economie regionali», Osservatorio Economico. Varaldo, R., (1979), (éd.), «Ristrutturazioni industriali e rapporti fra imprese», Franco Angeli, Milano.

Williamson, O., (1985), The Economic Institutions of Capitalism, The Free Press, New York. 